\title{
Sulfadiazine Sodium
}

National Cancer Institute

\section{Source}

National Cancer Institute. Sulfadiazine Sodium. NCI Thesaurus. Code C66566.

A sodium salt form of sulfadiazine, an intermediate-acting bacteriostatic, synthetic sulfanilamide derivative. 